\title{
Pyridylphosphonium Salts as Alternatives to Cyanopyridines in Rad- ical-Radical Coupling Reactions
}

\author{
Jacob W. Greenwood and Andrew McNally*
}

Department of Chemistry, Colorado State University, Fort Collins, Colorado 80523, United States

Radical Anions, Radical-Radical Coupling, Phosphonium Salts, BF 3 K Salts, Photoredox, Late-Stage Functionalization

ABSTRACT: Radical couplings of cyanopyridine radical anions represent a valuable technology for functionalizing pyridines, which are prevalent throughout pharmaceuticals, agrochemicals, and materials. Installing the cyano group, necessary for radical anion stabilization, is challenging and limits the use of this chemistry to simple cyanopyridines. We discovered that pyridyl phosphonium salts, installed regioselectively from $\mathrm{C}-\mathrm{H}$ precursors, are useful alternatives to cyanopyridines in radical-radical coupling reactions, expanding the scope of this reaction manifold to complex pyridines. Methods for both alkylation and amination of pyridines mediated by photoredox catalysis are described. Additionally, we demonstrate late-stage functionalization of pharmaceuticals, highlighting an advantage of pyridyl phosphonium salts over cyanopyridines.

Modern photoredox catalysis and electrochemistry have enabled new synthetic methods that proceed via open-shell intermediates. ${ }^{1}$ Under this regime, pyridine functionalization strategies have been developed where cyanopyridines undergo single-electron reduction to form dearomatized radical anions and related species that couple with other stabilized radicals (Scheme 1A). ${ }^{2}$ This reactivity is in stark contrast to other functional groups, such as halides, that eliminate after single-electron reduction and result in pyridyl radicals. ${ }^{3}$ Cyanopyridines have facilitated pyridine alkylation, allylation, and alkenylation reactions providing access to valuable building blocks for medicinal and agrochemical programs. ${ }^{4}$ The cyano group is essential for these methods, but a problem arises when applying this chemistry to complex pyridines, such as those found in pharmaceutical and agrochemical candidates. These structures are often devoid of pre-installed functional groups, and it is often challenging to selectively install a cyano group from $\mathrm{C}-\mathrm{H}$ precursors regioselectively. ${ }^{5}$ Furthermore, there are no reports of alternative functional groups that participate in radical anion coupling reactions in synthetically useful yields. We envisioned pyridyl phosphonium salts, regioselectively constructed from the $\mathrm{C}-\mathrm{H}$ bonds of a diverse set of pyridines, could serve as alternatives to cyanopyridines. ${ }^{6}$ Herein, we report couplings between both alkyl $\mathrm{BF}_{3} \mathrm{~K}$ salts and amines with pyridyl phosphonium salts, including late-stage functionalization of complex pyridine-containing pharmaceuticals using this strategy.

Recently, we reported a radical coupling reaction between a boryl-stabilized cyanopyridyl radical and a boryl-stabilized pyridylphosphonium radical. ${ }^{6 a}$ The intermediate radicals arose via an unusual inner-sphere process that would be difficult to extend to other coupling reactions. A significant advance would be to show pyridyl phosphonium salts functioning in broad reaction platforms, such as photoredox and electrochemical processes. We envisioned a redox-neutral alkylation reaction (Scheme 1B) via a radical coupling between radical anion $\mathbf{I}$, formed through single-electron reduction of a pyridylphosphonium salt $\left(E_{\mathrm{p} / 2}{ }^{\text {red }}=-1.51 \mathrm{~V}\right.$ vs. SCE $)$ and benzyl radical II, re-

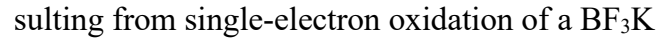

Scheme 1. Expansion of Radical Coupling Reactions to Complex Pyridines

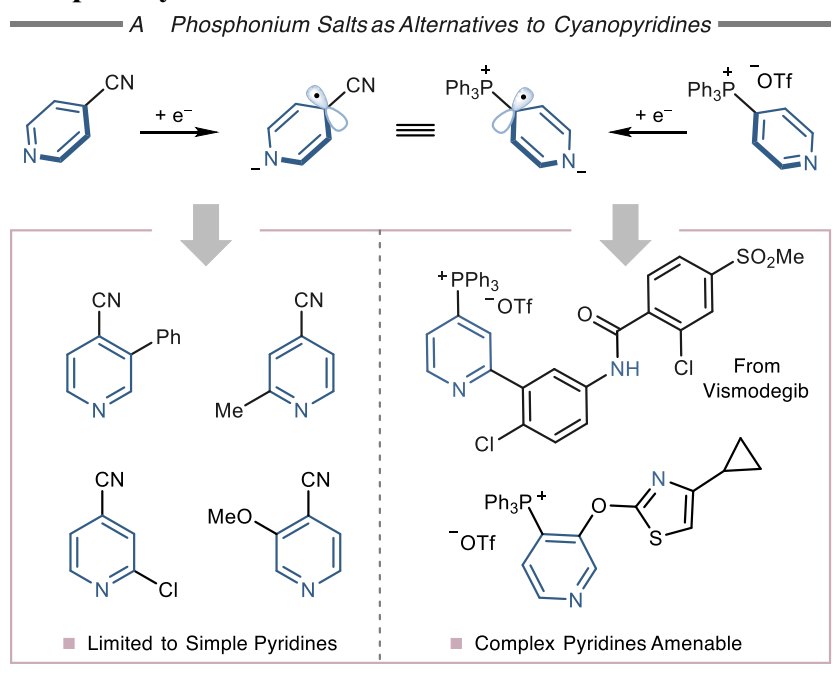

B Alkylation of Complex Pyridines via Phosphonium Salts

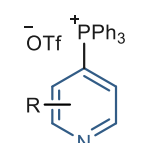<smiles>[R]C(Br)c1ccccc1</smiles>
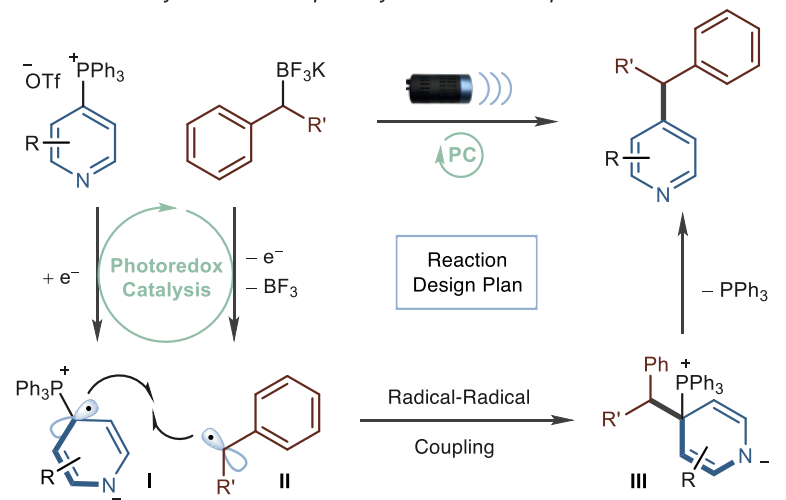

salt $\left(E^{\text {red }}=+1.10 \mathrm{~V}\right.$ vs. SCE for a primary benzylic salt $) .{ }^{7}$ Loss of triphenylphosphine from dearomatized intermediate (III) would furnish the alkylated pyridine product. Notably, the redox events could invert, where the photocatalyst oxidizes the 
$\mathrm{BF}_{3} \mathrm{~K}$ salt first and reduces the pyridylphosphonium salt second, broadening the scope of amenable photocatalysts.

We began our investigation by examining a series of photocatalysts for the coupling reaction of phosphonium salt 1a, formed with complete regioselectivity for the 4-position from 2-phenylpyridine, and benzylic $\mathrm{BF}_{3} \mathrm{~K}$ salt $\mathbf{2 a}$ under irradiation from a $455 \mathrm{~nm}$ Kessil light (Table 1A). We discovered that both $\operatorname{Ir}(\text { ppy })_{3}$ and $\left[\operatorname{Ir}\left(\mathrm{dF}\left(\mathrm{CF}_{3}\right) \text { ppy }\right)_{2}(\mathrm{dtbbpy})\right] \mathrm{PF}_{6}$ catalyze the transformation despite markedly different redox properties (entries $1-2) .{ }^{1 b}$ The Adachi-type photocatalyst 3DPAFIPN improved the yield to $77 \%$ with a further increase to $82 \%$ after increasing the reaction concentration (entries 3-4). Adding 2,6-lutidine, which has been shown as an effective additive for $\mathrm{BF}_{3} \mathrm{~K}$ cross-coupling reactions by the Molander group, had no impact on the yield of the model substrate (entry 5) but proved crucial in other cases. $^{8,9}$

We found that photocatalysts with redox potential windows unsuitable for this reaction were also competent (entries 67). ${ }^{1 b, 1 d}$ This led us to hypothesize that the transformation could also be promoted through an energy transfer mechanism, rather than single electron transfer. Supporting this theory, irradiating the reaction mixture with $455 \mathrm{~nm}$ light in the absence of photocatalyst led to trace amounts of product formation, but the same experiment with $365 \mathrm{~nm}$ light resulted in $66 \%$ yield of $\mathbf{3 a}$ (entries 8-9). These results suggest that exciting one of the coupling partners or an electron donor-acceptor (EDA) complex could also promote the reaction. UV-Vis spectroscopy did not indicate any evidence of an EDA complex, and we ascribe the reaction with $365 \mathrm{~nm}$ light to the overlap of the tails of the LEDs emission with the absorption of 1a at $345 \mathrm{~nm}$ (see Supporting Information). ${ }^{10}$ Based on these results and entries $1-6$, we considered that both electron- and energy-transfer pathways were viable mechanisms for the coupling process.

Using $\mathrm{Ru}(\mathrm{bpy})_{3}$ as a photocatalyst gave us further insight into the reaction mechanism (Table 1B). We did not observe any coupled product when we applied the catalyst alone, an expected result based on redox potentials. ${ }^{1 \mathrm{~b}}$ However, adding one equivalent of $\mathrm{BF}_{3} \cdot \mathrm{OEt}_{2}$ resulted in $47 \%$ yield of $\mathbf{3 a}$. From this study, we infer that Lewis acid coordination to pyridyl phosphonium salt 1a makes single-electron reduction more facile, enabling the reaction with the previously ineffective catalyst. Additionally, oxidizing salt $\mathbf{2 a}$ generates $\mathrm{BF}_{3}$ as the reaction progresses, so intermediate IV is likely part of the overall mechanistic scheme. Stern-Volmer quenching of 3DPAFIPN by pyridyl phosphonium salt 1 a occurs $\left(\mathrm{K}_{\mathrm{SV}}=14.9 \mathrm{M}^{-1}\right.$ and $\mathrm{K}_{\mathrm{q}}$ $=3.55 \times 10^{9} \mathrm{~L} \mathrm{~mol}^{-1} \mathrm{~s}^{-1}$ ), suggesting that radical anion formation occurs initially, but we acknowledge that a boryl-stabilized radical (IV) is also a likely coupling partner in the $\mathrm{C}-\mathrm{C}$ bond-forming step. ${ }^{11,12}$ Importantly, several reports of cyanopyridine radical coupling reactions have invoked an activation event to facilitate single electron reduction followed by radical coupling. ${ }^{2 b, 2 \mathrm{~g}, 2 \mathrm{i}, 13}$

Employing the optimized conditions, we investigated the scope of pyridylphosphonium salts in this coupling process (Table 2). Starting with building block-type pyridines, 2-substituted pyridines with electron-withdrawing and electron-donating groups couple effectively (3b-3d). Aryl and heteroaryl groups are also compatible at the 2-position (3e \& $\mathbf{3 f})$, and we did not observe any undesired reactivity
Table 1. Optimization of Pyridine Alkylation ${ }^{a}$ A - Reaction Optimization

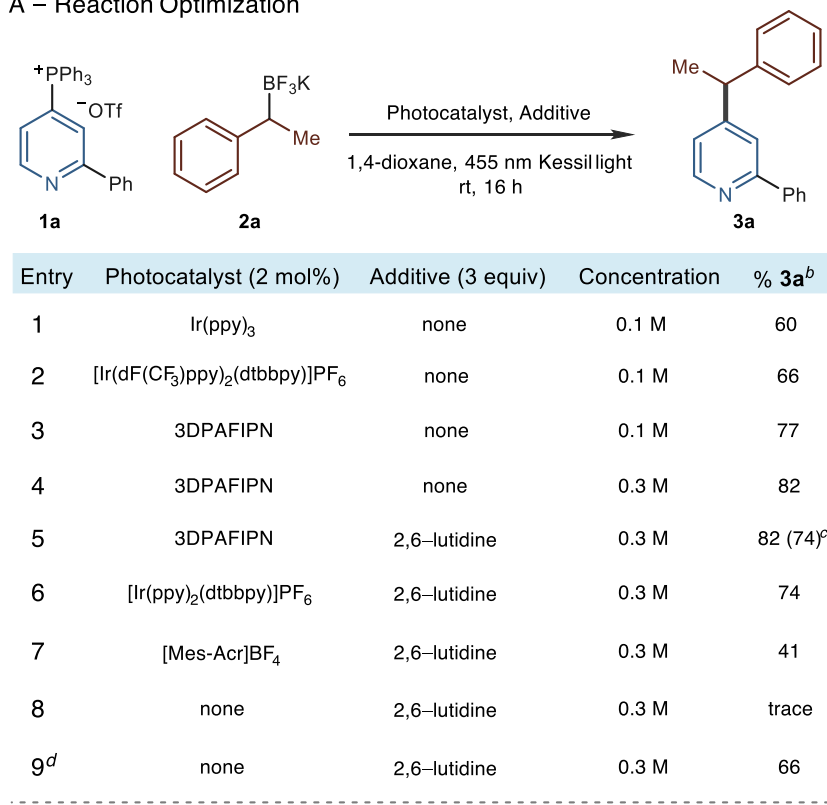

$\mathrm{B}-\mathrm{BF}_{3}$ Additive Enables Previously Ineffective Photocatalyst

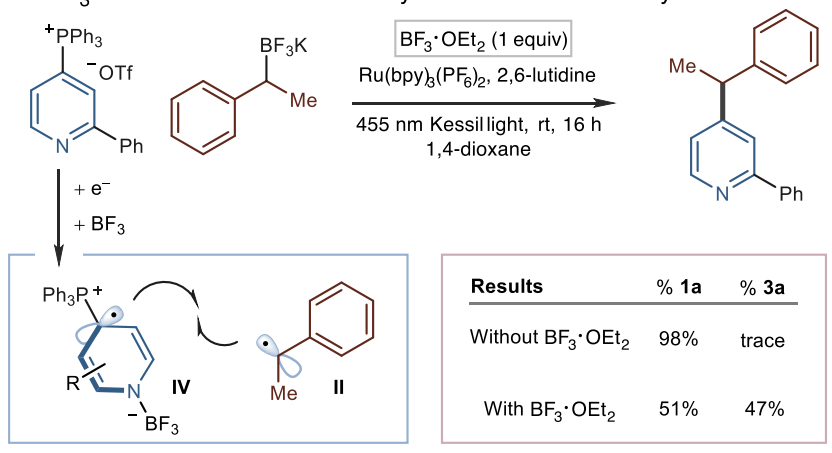

${ }^{a}$ Conditions: 1a (1.0 equiv), 2 a (2.0 equiv), photocatalyst ( $\left.2 \mathrm{~mol} \%\right)$, additive (3.0 equiv), rt. ${ }^{\text {b}}$ Yields determined by ${ }^{1} \mathrm{H}$ NMR analysis using 1,3,5-trimethoxybenzene as internal standard. 'Isolated yield in parenthesis. ${ }^{\mathrm{d}}$ Used $365 \mathrm{~nm}$ LEDs instead of $455 \mathrm{~nm}$ Kessil light for $89 \mathrm{~h}$.

of the carbon-iodine bond in $\mathbf{3 g}$. The reaction tolerates carbonbearing groups at the 3-position, although we did observe minor regioisomers in the crude ${ }^{1} \mathrm{H}$ NMR for pyridines $\mathbf{3 h}$ and $\mathbf{3 i}$. Disubstituted pyridines $\mathbf{3} \mathbf{j}-\mathbf{3 l}$, on the other hand, formed as single regioisomers in moderate to good yields.

Next, we converted a series of drug-like fragments and pharmaceuticals into phosphonium salts to evaluate in the alkylation reaction. These examples represent the most significant advantage of this chemistry as installing a cyano group would be challenging from the $\mathrm{C}-\mathrm{H}$ bond and limits the ability to make analog compounds. These structures contain multiple reactive sites and functional groups that could interfere with the coupling process. Nevertheless, we synthesized benzylated fragments $\mathbf{3 m - 3 q}$ without difficulty. Notably, other heterocycles are compatible, such as thiazoles and protected piperidines and pyrrolidines. The pyridine-pyrimidine biaryl 3o is particularly interesting as the phosphonium salt formed site-selectively on the pyrimidine ring and the photoredox coupling proceeded in good yield 
Table 2: Scope of Heterocyclic Phosphonium Salt Coupling Partners ${ }^{a}$
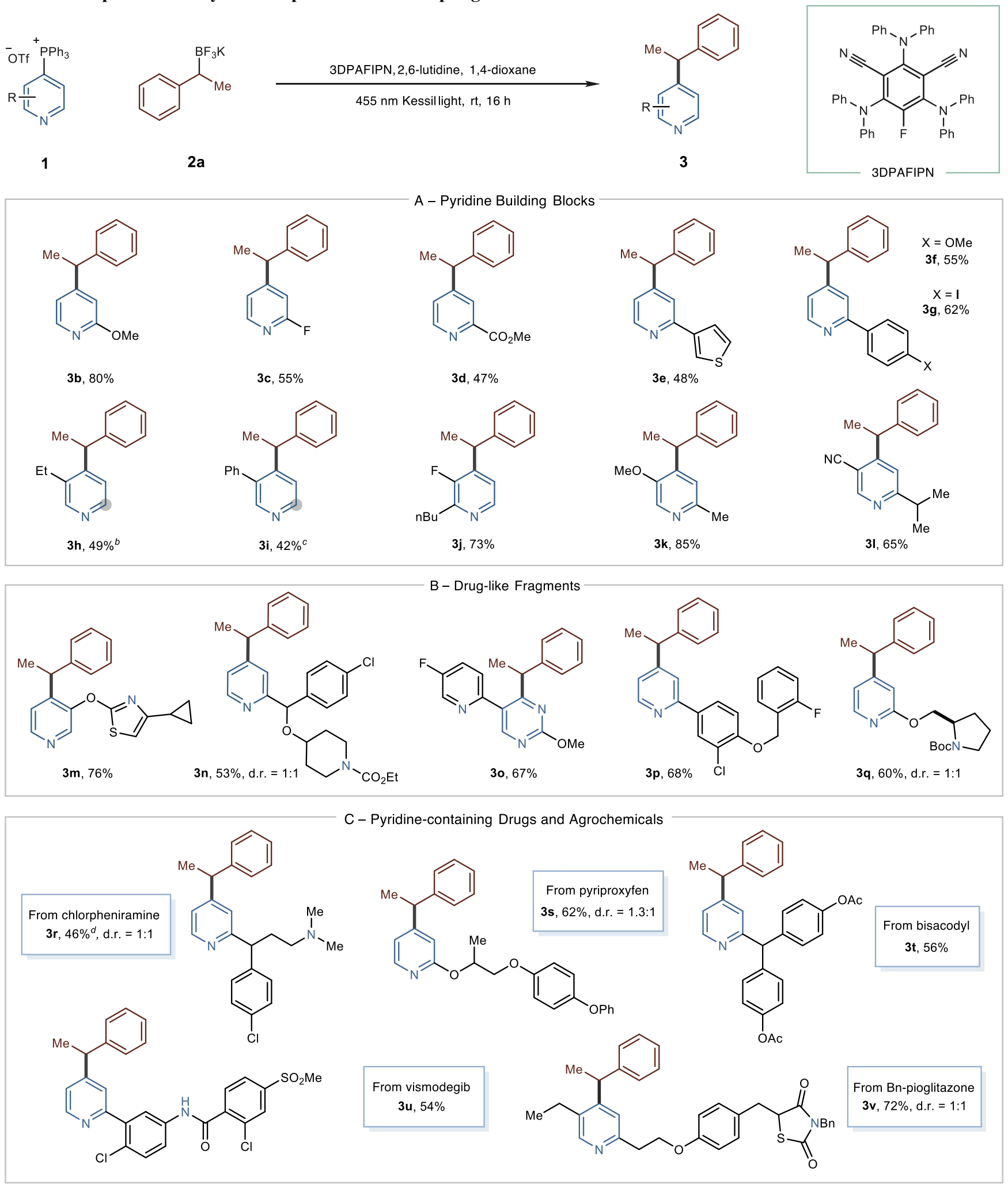

${ }^{a}$ Isolated yields of single regioisomers. Conditions: 1 (1.0 equiv), 2a (2.0 equiv), 3DPAFIPN (2 mol\%), 2,6-lutidine (3.0 equiv), 1,4-dioxane $(0.3 \mathrm{M})$, rt. ${ }^{b} 11: 1$ crude regioisomeric ratio. Isolated as single regioisomer. Grey circle denotes site of alkylation for minor regioisomer. ${ }^{c} 8: 1$ crude regioisomeric ratio. Isolated as single regioisomer. Grey circle denotes site of alkylation for minor regioisomer. ${ }^{d}$ With 1 equiv TfOH.

on this heterocycle. Lastly, we demonstrated coupling with four FDA-approved pharmaceuticals and an agrochemical that illustrate the functional group tolerance for protonated tertiary amines, amides, aryl halides, benzyl ethers, and sulfones (3r- 3v). These examples validate this tactic for late-stage functionalization of complex pyridines.

Scheme $2 \mathrm{~A}$ shows the scope of the $\mathrm{BF}_{3} \mathrm{~K}$ salts in the photoredox alkylation reaction. Secondary benzylic salts with 
Scheme 2: Scope of Radical Coupling Partners ${ }^{a}$
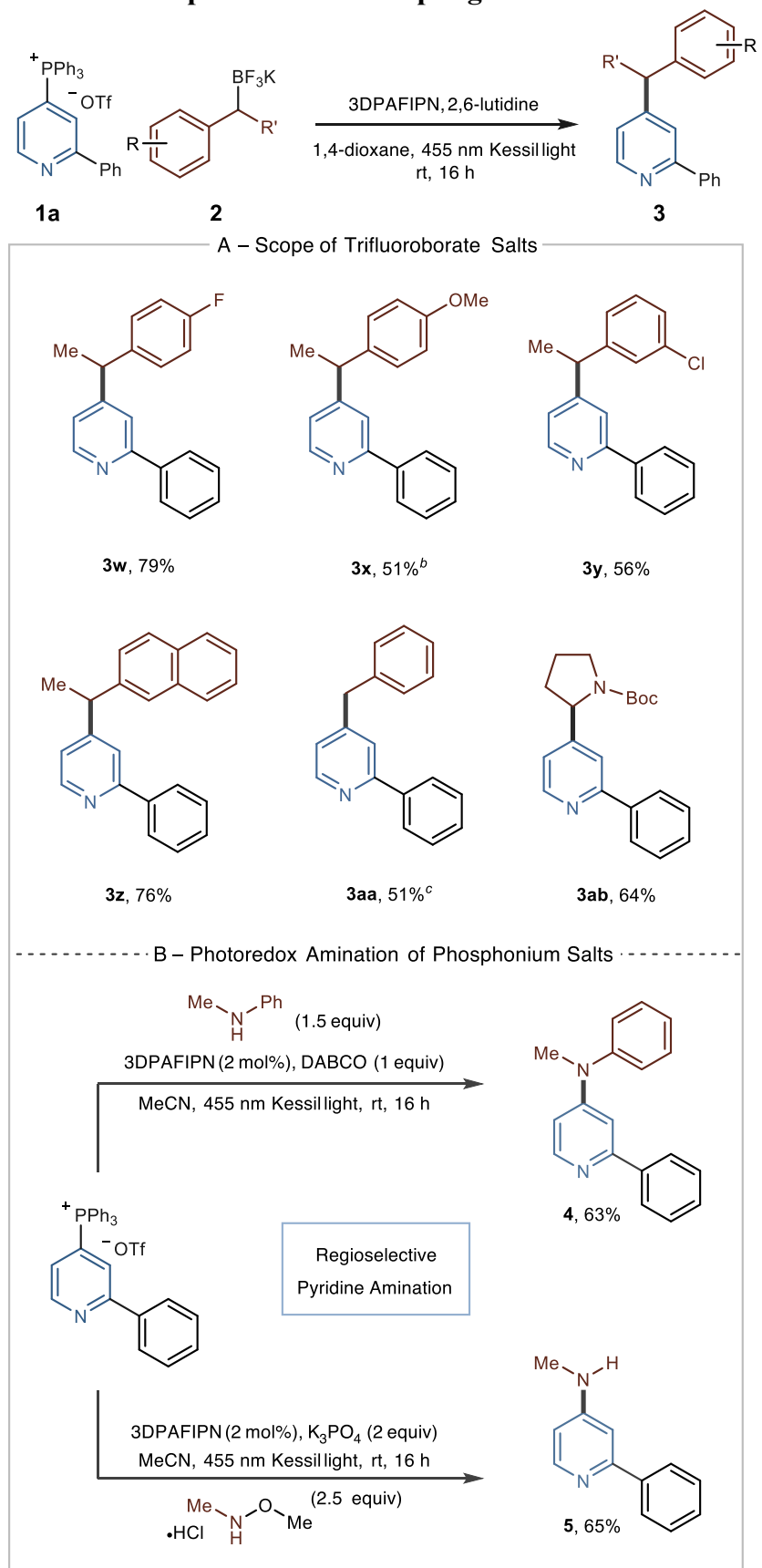

${ }^{a}$ Isolated yields of single regioisomers. Conditions: 1a (1.0 equiv), 2 (2.0 equiv), 3DPAFIPN ( $2 \mathrm{~mol} \%$ ), 2,6-lutidine (3.0 equiv), 1,4dioxane $(0.3 \mathrm{M}), \mathrm{rt} .{ }^{b} \mathrm{BF}_{3} \mathrm{~K}$ starting material is $1.2: 1$ mixture of regioisomers (benzylic:primary). ${ }^{c}>20: 1$ regioisomeric ratio and 5.7:1 mono:bis alkylated product in crude ${ }^{1} \mathrm{H}$ NMR spectrum. Isolated as single monoalkylated regioisomer.

electron-withdrawing and electron-donating groups are suitable coupling partners (3w-3y). In the case of $\mathbf{3 x}$, we added a 1.2:1 mixture of benzylic and homobenzylic $\mathrm{BF}_{3} \mathrm{~K}$ salts but only observed the benzylated product, presumably because the primary isomer is more difficult to oxidize. Secondary naphthyl and primary benzylic $\mathrm{BF}_{3} \mathrm{~K}$ salts are proficient, resulting in $\mathbf{3 z}$ and 3aa. The reaction also tolerates $\alpha$-amino $\mathrm{BF}_{3} \mathrm{~K}$ salts as evidenced by heterobenzylic amine derivative $\mathbf{3 a b}$.

Finally, we investigated pyridylphosphonium salts as alternatives to cyanopyridines in a recently reported amination reaction (Scheme 2B). Wu and coworkers published a method for photoredox-catalyzed $\mathrm{C}-\mathrm{N}$ bond formation that invoked cyano-stabilized pyridyl radicals as key intermediates. ${ }^{13}$ Applying pyridylphosphonium salt 1a to the reaction protocol with $N$ methyl aniline resulted in diaryl amine 4. Similarly, using $\mathrm{N}, \mathrm{O}$ dimethylhydroxylamine as a coupling partner, followed by in situ cleavage of the $\mathrm{N}-\mathrm{O}$ bond, formed aniline $\mathbf{5}$ in reasonable yield. Consistent with the results in Table 2, we expect that this reaction will be compatible with more complex pyridine phosphonium salts and further suggests that phosphonium ions can serve as surrogates for cyanopyridines in other radical anion coupling reactions.

In conclusion, we report that pyridylphosphonium salts behave as alternatives to cyanopyridines to extend the utility of radical-radical coupling reactions to more complex substrates. We showed that two distinct reactions, pyridine alkylation and amination, can proceed via phosphonium-stabilized radical intermediates. Our lab is currently investigating the capacity of pyridylphosphonium salts to participate in other open-shell reactions, as well as the mechanisms described in this study.

\section{ASSOCIATED CONTENT}

\section{Supporting Information}

The Supporting Information is available free of charge on the ACS Publications website.

\section{AUTHOR INFORMATION}

\section{Corresponding Author}

*Email: andy.mcnally@,colostate.edu

\section{ORCID}

Andrew McNally: 0000-0002-8651-163

Jacob W. Greenwood: 0000-0001-8512-2864

\section{Funding Sources}

This work was supported from the National Science Foundation under Grant No. (1753087).

\section{ACKNOWLEDGMENT}

We gratefully acknowledge the Shores and Miyake groups at CSU for their expertise and generous use of their equipment. We also acknowledge Benjamin Boyle (McNally group) for his assistance in preparing this manuscript.

\section{REFERENCES}

(1) (a) Twilton, J.; Le, C.; Zhang, P.; Shaw, M. H.; Evans, R. W.; MacMillan, D. W. C. The Merger of Transition Metal and Photocatalysis. Nat. Rev. Chem. 2017, 1, 0052. DOI: 10.1038/s41570017-0052. (b) Prier, C. K.; Rankic, D. A.; MacMillan, D. W. C. Visible Light Photoredox Catalysis with Transition Metal Complexes: Applications in Organic Synthesis. Chem. Rev. 2013, 113, 5322-5363. DOI: 10.1021/cr300503r. (c) Narayanam, J. M. R.; Stephenson, C. R. J. Visible Light Photoredox Catalysis: Applications in Organic Synthesis. Chem. Soc. Rev. 2011, 40, 102-113. DOI: 10.1039/B913880N. (d) Romero, N. A.; Nicewicz, D. A. Organic Photoredox Catalysis. Chem. Rev. 2016, 116, 1007510166. DOI: 10.1021/acs.chemrev.6b00057. (e) Horn, E. J.; Rosen, B. R.; Baran, P. S. Synthetic Organic Electrochemistry: An Enabling and Innately Sustainable Method. ACS Cent. Sci. 2016, 2, 302-308. DOI: $10.1021 /$ acscentsci.6b00091. 
(2) For selected examples, see: (a) McNally, A.; Prier, C. K.; MacMillan, D. W. C. Discovery of an $\alpha$-Amino C-H Arylation Reaction Using the Strategy of Accelerated Serendipity. Science 2011, 334, 1114-1116. DOI: 10.1126/science.1213920. (b) Hoshikawa, T.; Inoue, M. Photoinduced Direct 4-Pyridination of $\mathrm{C}\left(s p^{3}\right)-\mathrm{H}$ Bonds. Chem. Sci. 2013, 4, 3118. DOI: 10.1039/C3SC51080H. (c) Zuo, Z.; MacMillan, D. W. C. Decarboxylative Arylation of $\alpha$-Amino Acids via Photoredox Catalysis: A One-Step Conversion of Biomass to Drug Pharmacophore. J. Am. Chem. Soc. 2014, 136, 5257-5260. DOI: $10.1021 /$ ja501621q. (d) Cuthbertson, J. D.; MacMillan, D. W. C. The Direct Arylation of Allylic $s p^{3} \mathrm{C}-\mathrm{H}$ Bonds via Organic and Photoredox Catalysis. Nature 2015, 519, 74-77. DOI: 10.1038/nature14255. (e) Lima, F.; Kabeshov, M. A.; Tran, D. N.; Battilocchio, C.; Sedelmeier, J.; Sedelmeier, G.; Schenkel, B.; Ley, S. V. Visible Light Activation of Boronic Esters Enables Efficient Photoredox C $\left(s p^{2}\right)-\mathrm{C}\left(s p^{3}\right)$ Cross-Couplings in Flow. Angew. Chem. Int. Ed. 2016, 55, 14085-14089. DOI: 10.1002/anie.201605548. (f) Zhu, S.; Qin, J.; Wang, F.; Li, H.; Chu, L. Photoredox-Catalyzed Branch-Selective Pyridylation of Alkenes for the Expedient Synthesis of Triprolidine. Nat. Comm. 2019, 10, 749. DOI: 10.1038/s41467-019-08669-1. (g) Nicastri, M. C.; Lehnherr, D.; Lam, Y.; DiRocco, D. A.; Rovis, T. Synthesis of Sterically Hindered Primary Amines by Concurrent Tandem Photoredox Catalysis. J. Am. Chem. Soc. 2020, 142, 987-998. DOI: 10.1021/jacs.9b10871. (h) Ma, Y.; Yao, X.; Zhang, L.; Ni, P.; Cheng, R.; Ye, J. Direct Arylation of $\alpha$-Amino $\mathrm{C}\left(s p^{3}\right)-\mathrm{H}$ Bonds by Convergent Paired Electrolysis. Angew. Chem. Int. Ed. 2019, 58, 16548-16552. DOI: 10.1002/anie.201909642. (i) Lehnherr, D.; Lam, Y.; Nicastri, M. C.; Liu, J.; Newman, J. A.; Regalado, E. L.; DiRocco, D. A.; Rovis, T. Electrochemical Synthesis of Hindered Primary and Secondary Amines via ProtonCoupled Electron Transfer. J. Am. Chem. Soc. 2020, 142, 468478. DOI: $10.1021 /$ jacs.9b10870.

(3) (a) Aycock, R. A.; Wang, H.; Jui, N. T. A Mild Catalytic System for Radical Conjugate Addition of Nitrogen Heterocycles. Chem. Sci. 2017, 8, 3121-3125. DOI: 10.1039/C7SC00243B. (b) Seath, C. P.; Vogt, D. B.; Xu, Z.; Boyington, A. J.; Jui, N. T. Radical Hydroarylation of Functionalized Olefins and Mechanistic Investigation of Photocatalytic Pyridyl Radical Reactions. J. Am. Chem. Soc. 2018, 140, 15525-15534. DOI: $10.1021 /$ jacs.8b10238. (c) Boyington, A. J.; Seath, C. P.; Zearfoss, A. M.; Xu, Z.; Jui, N. T. Catalytic Strategy for Regioselective Arylethylamine Synthesis. J. Am. Chem. Soc. 2019, 141, 4147-4153. DOI: 10.1021 /jacs.9b01077. (d) Seath, C. P.; Jui, N. T. Intermolecular Reactions of Pyridyl Radicals with Olefins via Photoredox Catalysis. Synlett 2019, 30, 1607-1614. DOI: 10.1055/s-0037-1611527. (e) Singh, A.; Kubik, J. J.; Weaver, J. D. Photocatalytic C-F Alkylation; Facile Access to Multifluorinated Arenes. Chem. Sci. 2015, 6, 7206-7212. DOI: $\underline{10.1039 / C 5 S C 03013 G}$. (f) Senaweera, S.; Weaver, J. D. Dual CF, C-H Functionalization via Photocatalysis: Access to Multifluorinated Biaryls. J. Am. Chem. Soc. 2016, 138, 2520-2523. DOI: $10.1021 /$ jacs. 5 b 13450 .

(4) (a) Baumann, M.; Baxendale, I. R. An Overview of the Synthetic Routes to the Best-Selling Drugs Containing 6-Membered Heterocycles. Beilstein J. Org. Chem. 2013, 9, 2265-2319. DOI: 10.3762/bjoc.9.265. (b) Vitaku, E.; Smith, D. T.; Njardarson, J. T. Analysis of the Structural Diversity, Substitution Patterns, and Frequency of Nitrogen Heterocycles among U.S. FDA Approved Pharmaceuticals: Miniperspective. J. Med. Chem. 2014, 57, 10257-10274. DOI: 10.1021/jm501100b. (c) Eicher, T.; Hauptmann, S.; Speicher, A. The Chemistry of Heterocycles: Structure, Reactions, Syntheses, and Applications, 3rd ed.; Wiley-VCH: Weinheim, 2012.

(5) (a) Yu, X.; Tang, J.; Jin, X.; Yamamoto, Y.; Bao, M. ManganeseCatalyzed $\mathrm{C}-\mathrm{H}$ Cyanation of Arenes with $\mathrm{N}$-Cyano- $\mathrm{N}$-(4-methoxy)phenyl-p-toluenesulfonamide. Asian J. Org. Chem. 2018, 7, 550-553. DOI: 10.1002/ajoc.201700628. (b) Zhao, D.; Xu, P.;
Ritter, T. Palladium-Catalyzed Late-Stage Direct Arene Cyanation. Chem 2019, 5, 97-107. DOI:

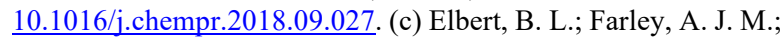
Gorman, T. W.; Johnson, T. C.; Genicot, C.; Lallemand, B.; Pasau, P.; Flasz, J.; Castro, J. L.; MacCoss, M.; Paton, R. S.; Schofield, C. J.; Smith, M. D.; Willis, M. C.; Dixon, D. J. C-H Cyanation of 6-Ring $N$-Containing Heteroaromatics. Chem. Eur. J. 2017, 23, 14733-14737. DOI: 10.1002/chem.201703931.

(6) (a) Koniarczyk, J. L.; Greenwood, J. W.; Alegre-Requena, J. V.; Paton, R. S.; McNally, A. A Pyridine-Pyridine Cross-Coupling Reaction via Dearomatized Radical Intermediates. Angew. Chem. Int. Ed. 2019, 58, 14882-14886. DOI: 10.1002/anie.201906267. (b) Koniarczyk, J. L.; Hesk, D.; Overgard, A.; Davies, I. W.; McNally, A. A General Strategy for Site-Selective Incorporation of Deuterium and Tritium into Pyridines, Diazines, and Pharmaceuticals. J. Am. Chem. Soc. 2018, 140, 1990-1993. DOI:

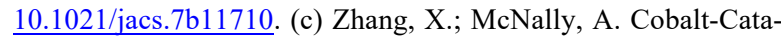
lyzed Alkylation of Drug-Like Molecules and Pharmaceuticals Using Heterocyclic Phosphonium Salts. ACS Catal. 2019, 9, 4862-4866. DOI: 10.1021/acscatal.9b00851. (d) Hilton, M. C.; Dolewski, R. D.; McNally, A. Selective Functionalization of Pyridines via Heterocyclic Phosphonium Salts. J. Am. Chem. Soc. 2016, 138, 13806-13809. DOI: 10.1021 /jacs.6b08662.

(7) Matsui, J. K.; Primer, D. N.; Molander, G. A. Metal-Free C-H Alkylation of Heteroarenes with Alkyltrifluoroborates: A General Protocol for $1^{\circ}, 2^{\circ}$ and $3^{\circ}$ Alkylation. Chem. Sci. 2017, 8, 35123522. DOI: $10.1039 / \mathrm{C} 7 \mathrm{SC} 00283 \mathrm{~A}$.

(8) (a) Tellis, J. C.; Primer, D. N.; Molander, G. A. Single-Electron Transmetalation in Organoboron Cross-Coupling by Photoredox/Nickel Dual Catalysis. Science 2014, 345, 433-436. DOI:

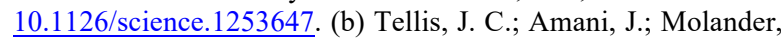
G. A. Single-Electron Transmetalation: Photoredox/Nickel Dual Catalytic Cross-Coupling of Secondary Alkyl $\beta$-Trifluoroboratoketones and -Esters with Aryl Bromides. Org. Lett. 2016, 18, 2994-2997. DOI: 10.1021/acs.orglett.6b01357. (c) Matsui, J. K.; Gutiérrez-Bonet, Á.; Rotella, M.; Alam, R.; Gutierrez, O.; Molander, G. A. Photoredox/Nickel-Catalyzed Single-Electron Tsuji-Trost Reaction: Development and Mechanistic Insights. $A n-$ gew. Chem. Int. Ed. 2018, 57, 15847-15851. DOI: 10.1002/anie.201809919.

(9) Examples of substrates that benefited from 2,6-lutidine additive: (a) The ${ }^{1} \mathrm{H}$ NMR yield of desired product decreased in the absence of 2,6-lutidine from $50 \%$ to $29 \%$ yield when switching from $\mathrm{BF}_{3} \mathrm{~K}$ salt 2a to potassium benzyltrifluoroborate. (b) The ${ }^{1} \mathrm{H}$ NMR yield of desired product decreased in the absence of 2,6-lutidine from $60 \%$ to $20 \%$ yield when switching from phosphonium salt 1 a to 1i. See Supporting Information for details.

(10) Lovett, G. H.; Chen, S.; Xue, X.-S.; Houk, K. N.; MacMillan, D. W. C. Open-Shell Fluorination of Alkyl Bromides: Unexpected Selectivity in a Silyl Radical-Mediated Chain Process. J. Am. Chem. Soc. 2019, 141, 20031-20036. DOI: 10.1021/jacs.9b11434.

(11) Cardinale, L.; Konev, M. O.; Jacobi von Wangelin, A. Photoredox-Catalyzed Addition of Carbamoyl Radicals to Olefins: A 1,4Dihydropyridine Approach. Chem. Eur. J. 2020, 26, 8239-8243. DOI: $\underline{10.1002 / \text { chem.202002410. }}$.

(12) Despite the similarities in photophysical properties between photocatalysts $\mathrm{Ru}(\mathrm{bpy})_{3}\left(\mathrm{PF}_{6}\right)_{2}$ and $\operatorname{Ir}(\mathrm{ppy})_{2}(\mathrm{dtbbpy}) \mathrm{PF}_{6}$, the iridium photocatalyst works well in the absence of $\mathrm{BF}_{3}$ additive while $\mathrm{Ru}(\mathrm{bpy}) 3\left(\mathrm{PF}_{6}\right)_{2}$ requires it. The reason for this is not yet understood and studies to explain this observation are ongoing.

(13) Zhou, C.; Lei, T.; Wei, X.-Z.; Ye, C.; Liu, Z.; Chen, B.; Tung, C.H.; Wu, L.-Z. Metal-Free, Redox-Neutral, Site-Selective Access to Heteroarylamine via Direct Radical-Radical Cross-Coupling Powered by Visible Light Photocatalysis. J. Am. Chem. Soc. 2020, 142, 16805-16813. DOI: $\underline{10.1021 / \text { jacs.0c07600. }}$. 
Phosphonium lons as Coupling Handles in Photoredox Processes
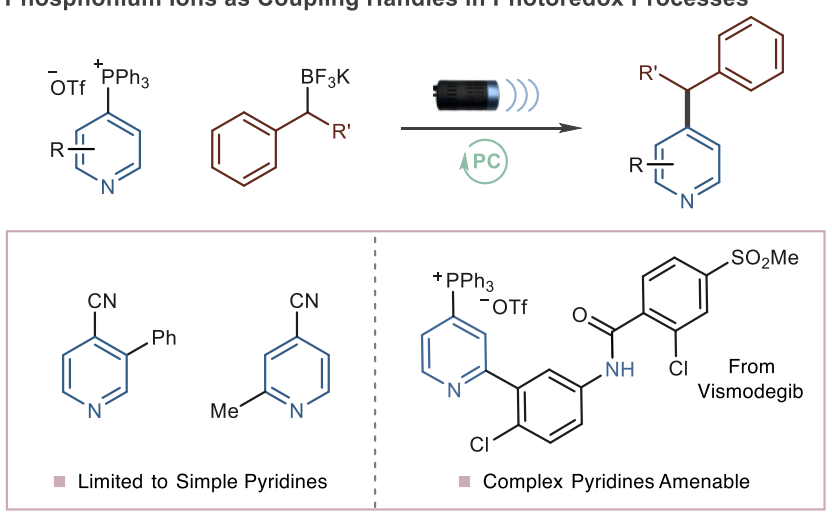